\title{
ANALYSES OF WOLF-RAYET SPECTRA
}

\author{
W.-R. HAMANN \\ Institut für Theoretische Physik und Sternwarte der Universität, \\ Olshausenstraße 40, D-2300 Kiel, Federal Republic of Germany
}

\section{Introduction}

Wolf-Rayet stars represent an important stage in the evolution of massive stars, but are only poorly understood so far. For a better knowledge one might determine their effective temperatures, luminosities and atmospheric compositions. But the emission-line dominated Wolf-Rayet spectra were not accessible to a quantitative analysis for a long time, because "standard" model calculations for static, plane-parallel stellar atmospheres are not adequate to that type of stars.

In a systematic approach to that problem we developed elaborate computer codes for non-LTE radiation transfer in expanding atmospheres (Hamann and Schmutz 1987; Wessolowski et al. 1988). Basic features of these models are briefly summarized in the following section. With this tool we then analyzed the helium line spectra of WR stars in our Galaxy and in the LMC (Sect. 3). A special study concerns the WR +0 eclipsing binary V444 Cygni (Sect. 4). In the next step we started to determine the hydrogen abundances in WN atmospheres (Sect. 5). Recently we proceeded to the next generation of models which include further elements, namely nitrogen (for WN stars) and carbon (WC stars), accounting for very complex model atoms and for non-grey radiative equilibrium (Sect. 6).

\section{The Model Atmospheres}

Prerequisite for the intended analyses of WR spectra is the adequate calculation of the non-LTE spectrum formation. We assume that the WR atmospheres are expanding, spherically symmetric, homogeneous and stationary. (Of course, the validity of these simplifying assumptions can only be justified a posteriori from the achieved agreement with the observation.) The equation of continuity then reads

$$
\dot{M}=4 \pi r^{2} \rho(r) v(r),
$$

where the mass-loss rate $\dot{M}$ is one of the essential free parameters of the models. The supersonic part of the velocity field is pre-specified by a law of the form

$$
v(r)=v_{\infty}\left(1-r_{0} / r\right)
$$

The "terminal velocity" $v_{\infty}$ is an adjustable parameter. At small velocities, a smooth transition towards static layers is ensured by suitable assumptions (for the definition of $r_{o}$ and other details see Hamann and Schmutz 1987). The "stellar radius" $R_{*}$, corresponding approximately to the location of the sonic point, is a further basic parameter which specifies a WR model.

The temperature structure is derived from the assumption of radiative equilibrium, until recently (cf. 
Sect. 6) evaluated in grey LTE approximation with the additional assumption that the temperature may never drop below $10 \mathrm{kK}$. A temperature parameter $\mathrm{T}$. is defined from the luminosity and the stellar radius $R$. by

$$
\mathrm{L}=4 \pi \sigma \mathrm{R}_{*}^{2} \mathrm{~T}_{*}^{4}
$$

and may be considered as an "effective temperature", related to the radius $R_{\star}$ of the hydrostatic stellar "core".

Hence a specific WR model is characterized by the four parameters $R_{*}, T_{*}, \dot{M}, v_{\infty}$, and by the chemical composition. Note that no self-consistent hydrodynamics are included in our semi-empiric models. Actually, not even the physical nature of the accelerating forces is known at present.

The radiation transfer in the spherically expanding atmosphere is treated in our code with a comoving frame formalism. The simultaneous solution of the equations of statistical equilibrium, which may represent very complex model atoms, is achieved by "iteration with approximate lambda operators" (cf. Hamann 1985, 1986, 1987). This new technique allows for the first time to treat such formidable non-LTE problems.

\section{Analyses with Pure-Helium Models}

In a first stage we worked with models which only account for pure helium. For a number of galactic WN stars we performed detailed fits of the helium line profiles and of the continuous energy distribution. A typical example was presented by Hamann et al. (1988), concerning the WN5 star HD50896 alias WR6. Meanwhile we have performed eight "fine analyses" of that kind, and we always arrived at the following conclusions. The models can reproduce well the general features of the observed helium lines. The theoretical continua are always in good agreement with observation, when accounting for the interstellar reddening. Hence our models are basically adequate to describe real WR atmospheres. However, some discrepancies in details of the line profiles might indicate that the models are not yet perfect.

Encouraged by these considerations, we established an extensive grid of models which now allows to determine the parameters of various WR stars with little effort. (For a "coarse analysis" it is sufficient to account for equivalent widths only, instead of a detailed line-profile fitting.) 30 galactic WR stars, covering the different subtypes, have been analyzed in this way (Schmutz et al. 1989). Fig. 1 (open symbols) gives the positions of the WN stars in the HR diagram. We find that the stellar temperatures and luminosities are smaller than most authors had expected previously. It turned out that there is no simple correlation between subtype (e.g. WN5, WN6 ...) and T., i.e. a one-dimensional classification is obviously insufficient. For instance, some of the WNE-A stars (i.e. early-type WN with weak lines) are actually as "cool" as WNL ("late") types, namely $\approx 35 \mathrm{kK}$.

From the theoretical models we learned that models with the same ratio $R_{*} / \dot{\mathrm{M}}^{2 / 3}$ show very similar spectra. Consequently, the unique determination of stellar radius and mass-loss rate requires the knowledge of one distance-dependent quantity, e.g. the absolute visual magnitude $\mathrm{M}_{\mathrm{v}}$. Fortunately the distances of many WR stars are known from cluster or association memberships, but for individual stars this assignment may be in error.

For WR stars in the Large Magellanic Cloud the absolute magnitudes are more reliable, because their uniform distance is known and the interstellar reddening is generally small. Moreover, it is interesting to compare their parameters with the galactic members. Therefore we extended our analysis of WN stars to the LMC (Koesterke et al., in preparation). Spectra were obtained with the ESO 3.6m telescope and EFOSC. The analysis of the helium lines is performed in the same way and with the same grid of models as used for the galactic stars. Thus the comparison should be unbiased from systematic errors of the models. The - still somewhat preliminary - results for the LMC stars are also indicated in 


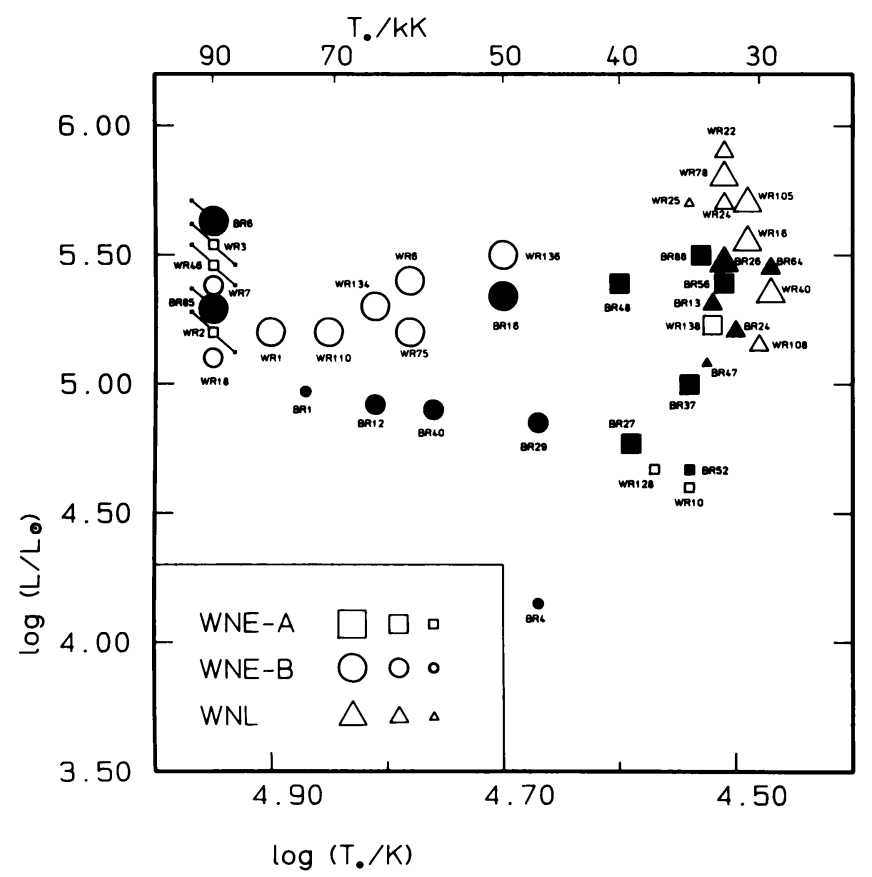

Fig 1. Positions of WN stars in the HR diagram, as obtained by analyses with pure-helium models. The individual stars are identified by their catalogue number (van der Hucht et al., 1981; Breysacher, 1981). LMC stars (filled symbols) tend to smaller luminosities than galactic WN stars (open symbols). Different subtypes are distinguished by different symbols, as explained in the inlet. The different sizes of the symbols indicate the mass loss rate, depending on whether $\log \left\{\dot{M} /\left(\mathrm{M}_{\odot} / \mathrm{yr}\right)\right\}$ is larger than $-\mathbf{4 . 2}$ (large symbols), smaller than -4.8 (small symbols) or in between (medium size).

the HR diagram (Fig. 1, filled symbols). The comparison with the galactic WN stars reveals that, on the average, the LMC stars lie at lower luminosities. Strikingly different is the position of the early-type WN stars with strong lines. Among our sample we find five such WNE-B stars in the LMC with luminosities below $10^{5} \mathrm{~L}_{\odot}$ which have no galactic counterparts. But, of course, we must be cautious with statistical conclusions because our program stars are selected arbitrarily.

\section{The Eclipsing Binary V444 Cygni (WN5 + 06?)}

The binary system V444 Cygni provides a crucial test of our WR models. We synthesize the light curve, accounting for the mutual eclipse of the extended WR atmosphere and the $O$ star. The former is given by our models, i.e. we neglect any disturbance of its spherical symmetry by the companion. The basic result is that we can reproduce the observed light curve of V444 Cygni with our model, but the solution is not unique (Schwarz et al., in preparation; Hamann et al., these proceedings; Hamann et al., 1990).

We can also satisfactorily fit the helium lines in the observed composite spectrum. The stellar parameters of such a spectral analysis depends, however, on the brightness ratio between the binary components which is a free parameter. We have not yet succeeded in fitting both, the composite spectrum and the light curve, with exactly the same set of parameters, but this point must still be further investigated. 


\section{Hydrogen Abundances in WN Stars}

The most comprehensive study about hydrogen abundances in WN stars was performed by Conti et al. (1983). Their results confirmed that WN atmospheres consist predominantly of helium, while hydrogen is more or less depleted or even absent within the detection limit. A histogram (Fig. 12 in Conti et al. 1983) illustrates that there is no clear correlation between hydrogen detection and spectral subtype: stars with hydrogen and stars without detectable hydrogen are found among both, the WNE ("early") as well as the WNL ("late").

This picture drastically changes when the hydrogen detection is not correlated with the subtype, but instead with the stellar parameters as obtained by our spectral analyses. Fig. 2 shows a clear separation between the two groups: the "cool" stars with $\mathrm{T}_{*} \approx 35 \mathrm{kK}$ exhibit hydrogen, while the "hot" stars $\left(\mathrm{T}_{*}>\right.$ $60 \mathrm{kK}$ ) do not. WR136 is intermediate in temperature and in hydrogen abundance. The only exception, WR3, is a peculiar object in many aspects. Note that four of the stars classified as WNE-A (WR10, WR128, BR52, BR88) are actually "cool", and just these stars show hydrogen.

Conti et al. (1983) derived the hydrogen abundances from a "semiquantitative" study of the BalmerPickering decrement. We now repeat this analysis with our detailed model calculations in order to obtain more precise results (Hamann et al., these proceedings; Hamann et al., 1990). Up to now we have completed four fine analyses of the described kind. In one case (WR22) the hydrogen mass fraction reaches $40 \%$, while for the other two "cool" stars $\left(\mathrm{T}_{*}<35 \mathrm{kK}\right)$ the hydrogen mass fraction is of the order of $B_{H}=20 \%$. WR136, which is considerably hotter $\left(T_{*} \approx 50 \mathrm{kK}\right)$, has only $6 \%$ hydrogen, and thus appears as a link to the "hot" WN stars in which hydrogen is not detectable (cf. Fig. 2).

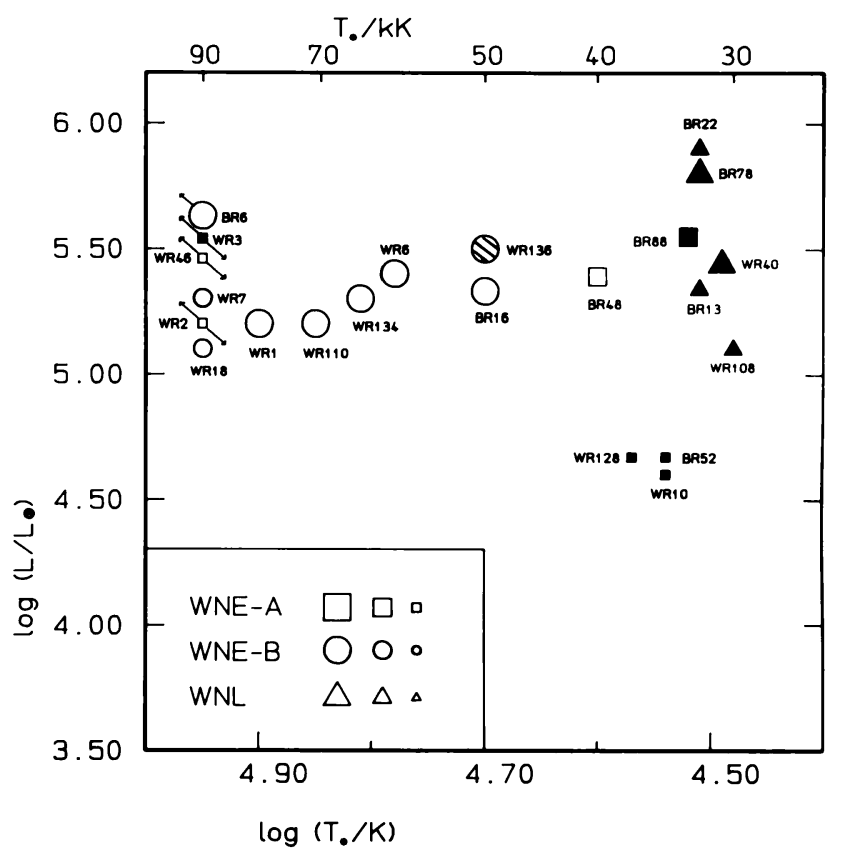

Fig. 2. Hydrogen in WN stars. The individual stars are identified as in Fig. 1. Open symbols denote stars without hydrogen, filled symbols stars with hydrogen detected (according to Conti et al. 1983). WR136 got a hatched symbol because Conti et al. could not detect any hydrogen, while we found a very small abundance $\left(B_{H}=6 \%\right)$. The stellar parameters $(L, T$.) are from our spectral analyses (Schmutz et al. 1989, partly improved from "fine analyses"; Koesterke et al., in preparation). Different subtypes are distinguished by different symbols, as explained in the inlet. The different size of the symbols indicates the massloss rate: $\log \left\{\dot{M} /\left(\mathrm{M}_{0} / \mathrm{yr}\right)\right\}>-4.2$ (large symbols), $<-4.8$ (small symbols), or in between (medium size). 


\section{WR Models with Nitrogen and Carbon}

The non-LTE models for WR atmospheres reach their next stage of sophistication when not only helium (and possibly hydrogen), but also the most important "metals" are taken into account. Hillier (1988) was the first who introduced nitrogen into his calculations, and meanwhile he proceeded to carbon in order to synthesize WC spectra (Hillier, 1989).

Our recent models now also include nitrogen, represented by a model atom with 90 levels and about 1500 lines, 350 of them treated explicitely. Low-temperature dielectronic recombination via hundreds of transitions is allowed. Together with the nitrogen we introduced an improved temperature structure which now fully accounts for radiative equilibrium, instead of the grey LTE approximation applied so far. Test calculations with these improved models (Hamann and Wessolowski, 1989), setting the nitrogen abundance to $1.5 \%$ by mass, show no significant reaction of the helium spectrum. Thus the previous results obtained from models with pure helium and "grey" temperature structure remain valid, as far as WN subtypes are concerned.

As our next step we try to reproduce in detail the nitrogen line spectrum of one WN star (WR 136; Wessolowski et al., these proceedings). From preliminary results we conclude that some general agreement can be achieved, while remaining discrepancies can be attributed to problems with the very complex nitrogen model atom.

Calculations for WC stars which account for a complex carbon model atom are also available now in our group (Leuenhagen et al., in preparation). A first analysis of WR111 confirms the results of Hillier (1989), especially concerning the carbon mass fraction of about one half. As the carbon opacities control the radiative transfer, the analysis of WC spectra with pure-helium models (Schmutz et al., 1989) could only be preliminary. The improved models with carbon now yield, e.g., for WR111 a stellar temperature of about $60 \mathrm{kK}$, instead of $35 \mathrm{kK}$ obtained previously. Therefore we will refrain from discussing the parameters of WC stars until we have analyzed more of them with the adequate models.

\section{Conclusions: the Evolutionary Status of Wolf-Rayet Stars}

The spectral analyses indicate that most WR stars are considerably cooler and less luminous than predicted by the standard evolutionary calculations, which generate WR stars as a post-red-supergiant stage (cf. Fig. 3). The hydrogen abundances in WN stars provide a further check of the evolutionary szenario. The most luminous WNL star in our sample (WR22) has about $40 \%$ hydrogen, indicating that this star has either not yet evolved to the red, or - as it is known as a spectroscopic binary - was formed by mass transfer. For the two other "cool" WN stars $\left(\mathrm{T}_{*} \approx 35 \mathrm{kK}\right)$, irrespective whether classified as WNL or WNE-B, we find typical hydrogen mass fractions of about $20 \%$, just as predicted for the phase of rapid blueward post-RSG evolution. The observed disappearance of hydrogen towards higher stellar temperatures also agrees qualitatively with the theoretical tracks.

The agreement with the evolutionary calculations ends, however, when the stellar parameters are considered. The observed luminosities are smaller than predicted by more than a factor of ten. Very small luminosities are determined for a couple of WNE-B stars in the LMC, possibly indicating that under low-metallicity conditions even less massive stars can reach the WN phase, compared to our Galaxy. Recall that the ratio between the number of WN stars and WC stars also strongly differs between the Galaxy and the LMC.

Summarizing, we state that we can perform now a quantitative analysis of Wolf-Rayet spectra. For a large number of stars we already obtained stellar parameters and atmospheric abundances, and further results are to be expected soon. This empirical basis strongly constrains any evolutionary explanations. Hopefully it will help us to a better understanding of the evolution of massive stars. 


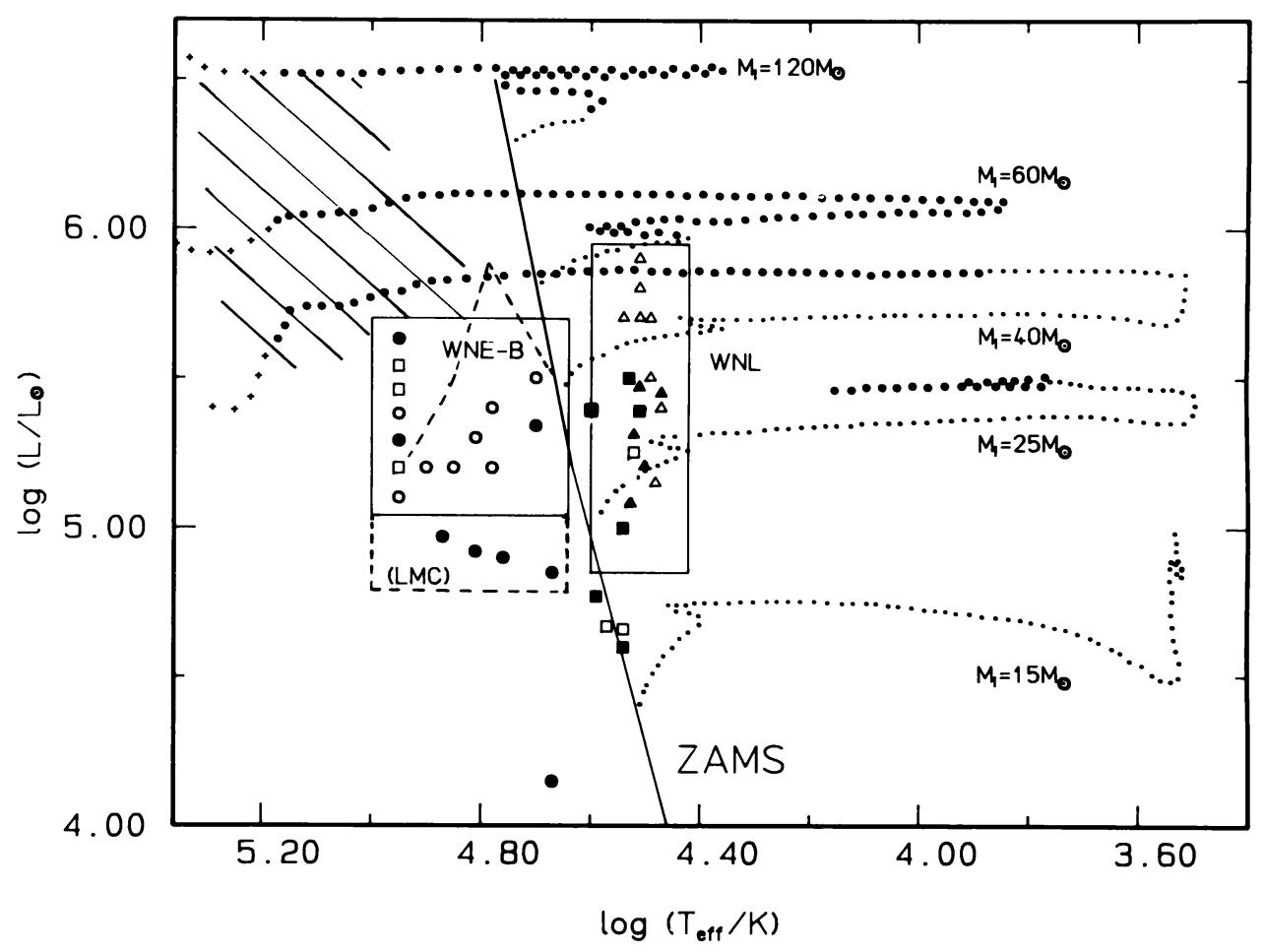

Fig 3. Loci of WR stars in the HR diagram, as obtained from spectral analyses. Different symbols denote the subgroups WNL $(\Delta)$, WNE-A $(\square)$, and WNE-B $(O)$, while open or full symbols indicate whether the stars belong to our Galaxy or the LMC, respectively. The overall location of the WNL and the WNE-B subgroups is additionally emphasized by large boxes. The box for the WNE-B stars in the LMC (broken line) extends to smaller luminosities than for our galactic sample. Superimposed are evolutionary tracks (from Maeder and Meynet, 1987), with indication of the predicted surface abundances: ... "normal", ... WN-type, +++ WC-type. Only in the hatched region the evolution proceeds slowly enough so that WR stars are likely to be observed.

\section{References}

Breysacher, J.: 1981, Astr. Ap. Suppl. Ser. 43, 203

Conti, P.S., Leep, E.M., Perry, D.N.: 1983, Ap. J. 268, 228

Hamann, W.-R.: 1985, Astr. Ap. 148, 364

Hamann, W.-R.: 1986, Astr. Ap. 160, 347

Hamann, W.-R.: 1987, in "Numerical Radiative Transfer", ed. W. Kalkofen, Cambridge University Press, p. 35

Hamann, W.-R., Schmutz, W.: 1987, Astr. Ap. 174, 173

Hamann, W.-R., Schmutz, W., Wessolowski, U.: 1988, Astr. Ap. 194, 190

Hamann, W.-R., Wessolowski, U.: 1990, Astr. Ap. 227, 171

Hamann, W.-R., Wessolowski, U., Schwarz, E., Dü nnebeil, G., Schmutz, W.: 1990, in "Properties of Hot Luminous Stars: Boulder-Munich Workshop", ed. C. D. Garmany, The A.S.P. Conference Series, Vol. 7, p. 259

Hillier, D.J.: 1988, Ap. J. 327, 822

Hillier, D.J.: 1989, Ap. J. 347, 392

van der Hucht, K.A., Conti, P.S., Lundström, I., Stenholm, B.: 1981, Space Sci. Rev. 28, 227

Maeder, A., Meynet, G.: 1987, Astr. Ap. 182, 243

Schmutz, W., Hamann, W.-R., Wessolowski, U.: 1989, Astr. Ap. 210, 236

Wessolowski, U., Schmutz, W., Hamann, W.-R.: 1988, Astr. Ap. 194, 160 